\title{
miR-15a-5p targets PHLPP2 in gastric cancer cells to modulate platinum resistance and is a suitable serum biomarker for oxaliplatin resistance
}

\author{
K. PANG, J. SONG, Z. BAI, Z. ZHANG* \\ ${ }^{1}$ Department of General Surgery, Beijing Friendship Hospital, Capital Medical University, Xicheng District, Beijing, China \\ ${ }^{*}$ Correspondence: zhangzht@ccmu.edu.cn
}

Received May 21, 2018 / Accepted July 22, 2019

\begin{abstract}
MicroRNAs can bind with target genes thus inhibiting their expression levels to regulate cell survival, hence serve as serum biomarkers for a variety of purposes. Our aim was to explore the role of miR-15a-5p in the cisplatin/oxaliplatin resistance of gastric cancer. In this study, the growth and apoptosis of gastric cancer cell lines were measured with cell viability assay and flow cytometry, respectively. Dual-luciferase assay was applied for miRNA target validation. Expression of PHLPP2 and miR-15a-5p were measured by quantitative polymerase chain reaction and western blot, respectively. Serum miR-15a-5p level of 82 gastric cancer patients was examined by qPCR. Our results indicated that miR-15a-5p overexpression increased cisplatin resistance of gastric cancer cells, whereas miR-15a-5p downregulation decreased the resistance. miR-15a-5p directly targets and inhibits PHLPP2 in gastric cancer cells, enhancing downstream Akt phosphorylation. Moreover, overexpression of miR-15a-5p could attenuate the decrease in cisplatin resistance induced by PHLPP2 overexpression. Additionally, we observed that lower serum miR-15a-5p level was significantly correlated with better response to oxaliplatin-based chemotherapy as well as better 3-year survival. In conclusion, miR-15a-5p was recognized as a biomarker for platinum resistance and prognosis in gastric cancer, interference of which may be a promising strategy for sensitizing gastric cancer to platinum drugs.
\end{abstract}

Key words: gastric cancer, platinum resistance, miR-15a-5p, PHLPP2, biomarker

Gastric cancer is the third lethal cancer amongst all types of cancers considering its high mortality rate [1], for which surgery in combination with chemotherapy is a major solution. Platinum drugs (mainly cisplatin and oxaliplatin) are among the therapeutic drugs most widely used in chemotherapy against gastric cancer. It binds with DNA and renders cross-links, consequently inducing cell apoptosis [2]. Regarding patients with gastric cancer in the advanced stage, multi-drug combinational chemotherapies that include platinum could extend survival time and increase response rates $[3,4]$. However, even though initial responses are often expectable, acquired chemoresistance often leads to the final failure of treatment.

MicroRNAs (miRNAs) are small non-coding RNAs that consist of 20 or so nucleotides. They function mainly through downregulating the expression of their target genes [5]. miRNAs complementarily bind to target sequences of the 3'-untranslated regions of their downstream genes, prompting the formation of RNA-induced silencing complex (RISC), subsequently causing mRNA degradation and inhibition of the translation of corresponding proteins [6].
Like other non-coding RNAs, miRNAs are mostly expressed in cells. However, a trace amount of miRNAs can also be detected in the extracellular environment that is almost all body fluid, including serum. Cellular miRNAs, once enter the extracellular environment, degrade within seconds [7], whereas extracellular miRNAs, or specifically serum miRNAs, are remarkably stable despite the varying temperature, $\mathrm{pH}$, and extended times of freeze-thaw cycles. For this interesting property, numerous studies have focused on the potential of serum miRNAs as biomarkers for the diagnosis and prognosis of malignant tumors in recent years.

In our previous works, we identified several differentially expressed miRNAs between a gastric cancer cell line and its cisplatin-resistant cell line by sequencing, of which miR-15a-5p turned out to be among the most significant one. Since miRNAs function mainly by inhibiting the expression of its target genes, in this study, we revealed that PHLPP2 was a novel target of $\mathrm{miR}-15 \mathrm{a}-5 \mathrm{p}$ in the regulation of platinum resistance of gastric cancer.

PHLPP2 is characterized as a member of the PHLPP family that suppresses the progression of the cell cycle, as 
well as enhances apoptosis among assorted types of carcinoma, and it is proven to have a suppressive effect on the $\mathrm{PI} 3 \mathrm{~K} / \mathrm{AKT}$ pathway [8]. In this research, we found that miR-15a-5p overexpression significantly decreases the sensitivity of MGC803 cells to cisplatin by enhancing the viability of cells and reducing apoptosis through interacting with PHLPP2 and subsequent phosphorylation of AKT. Moreover, the significant correlation between serum miR-15a-5p level and the response toward oxaliplatin-based chemotherapy of gastric cancer patients made us believe that miR-15a-5p might serve as a biomarker for platinum resistance and prognosis in gastric cancer.

\section{Materials and methods}

Culture of cell lines. MGC803 cell line was purchased from the National Infrastructure of Cell Line Resource of China. The cisplatin-resistant cell line of MGC803 (MGC803/ DDP) was established in our laboratory. For creating a cisplatin-resistant cell line, MGC803 was exposed to gradient concentration of cisplatin (Merck, Darmstadt, Germany) till the concentration reached $1 \mu \mathrm{g} / \mathrm{ml}$, followed by intermittent culture of cisplatin at this concentration for 1 year. DMEM culturing medium (Gibco, NY, USA) with 10\% fetal bovine serum (Gibco), penicillin and streptomycin (Solarbio, Beijing, China) were used in the culture of both cell lines. Cells were cultured in a $37^{\circ} \mathrm{C}, 5 \% \mathrm{CO}_{2}$ incubator (Thermo Fisher, Waltham, USA).

Cell growth and cytotoxicity assay. For cell growth assay, MGC803 and MGC803/DDP cells in the logarithmic phase were seeded to 96-well plates with a density of 2000 cells per well in triplicate. Cell Counting Kit 8 (CCK-8) (Thermo Fisher) solution was added into each well at $24,48,72$, and $96 \mathrm{~h}$ of culture. After incubation in a $\mathrm{CO}_{2}$ incubator at $37^{\circ} \mathrm{C}$ for $1 \mathrm{~h}$, the values of absorbance in each well were read at 450 nm on an enzyme-labeled instrument (Thermo Fisher). For cytotoxicity assay, 96-well plates were used for cell seeding and 5000 cells were seeded in each well. After $24 \mathrm{~h}$ incubation, cisplatin (Sigma, St. Louis, USA) with different concentrations at $0,2,4,8,16$, and $32 \mu \mathrm{g} / \mathrm{ml}$ was added. Then after $48 \mathrm{~h}$ incubation, cell viabilities were assessed with the CCK-8 assay as mentioned above.

Flow cytometry analysis of apoptosis. An eBioscience $^{\mathrm{TN}}$ Annexin V-FITC Apoptosis Detection Kit (Invitrogen, Carlsbad, USA) is employed for cell apoptosis assay. Apoptosis analysis was conducted with a flow cytometer (BD Biosciences, Franklin Lakes, USA). FlowJo (vX.0.7) software was applied in the data processing.

cDNA synthesis and quantitative PCR. Trizol (Invitrogen, Carlsbad, USA) was applied in total RNA extraction; Reverse transcription was conducted with M-MLV Reverse Transcription system (Takara, Dalian, China). miRNA from cells was extracted with EasyPure miRNA Kit (Transgen, Beijing, China). miRNeasy Serum/Plasma Kit (Qiagen, Düsseldorf, Germany) was utilized in serum miRNA extrac- tion; Mir- $\mathrm{X}^{\mathrm{T}}$ miRNA First-Strand Synthesis Kit (Takara, Dalian, China) was applied in cDNA synthesis. Quantitative real-time PCR was conducted with 7500 FAST realtime PCR System (Applied Biosystems, Foster City, USA). Internal controls for total RNA and miRNA from cells were GAPDH and U6 respectively. Cel-miR-39 was used as an external control for miRNA from serum. Source of primers: miR-15a-5p and U6 (Takara, Dalian, China), GAPDH and PHLPP2 (Sangon, Shanghai, China), cel-miR-39 (Ribobio, Guangzhou, China).

Plasmid and miRNA negative control (NC)/precursor/ inhibitor transfection. MGC803 and MGC803/DDP cells were seeded into $60 \mathrm{~mm}$ dishes and transfected with $50 \mathrm{nM}$ Pre-miR ${ }^{\mathrm{mm}}$ miRNA Precursor Negative Control \#1 (miR-NC), Pre-miR ${ }^{\mathrm{m}}$ miRNA Precursor of miR-15a-5p (miR-15a-5p precursor), or Anti-miR ${ }^{\mathrm{m}} \mathrm{miRNA}$ Inhibitor of miR-15a-5p (miR-15a-5p inhibitor) (Thermo Fisher) in triplicates via reverse transfection. As with plasmid, PHLPP2 sequence (Invitrogen, Carlsbad, USA) was cloned onto pcDNA3.0 vectors (Invitrogen, Carlsbad, USA). Lipofectamine 3000 transfection system (Invitrogen, Carlsbad, USA) was used for the transfection.

Western blotting. Cell harvest was conducted with Cell Lysis Buffer (CST, Danvers, USA). Protein separation was performed with $8 \%$ SDS/polyacrylamide gels before transferring onto nitrocellulose membranes (CST, Danvers, USA), which was subsequently blocked for $1 \mathrm{~h}$ using $5 \%$ non-fat dried milk dissolved with TBST. After blocking, the membranes were incubated in $4{ }^{\circ} \mathrm{C}$ overnight with primary antibodies: anti-PHLPP2 (1:3000, ab71973, Abcam), anti-Akt (1:1000, 4691S, CST), anti-pAkt (1:2000, 4060S, CST), and anti-GAPDH (1:1000, 5174S, CST). The next morning, membranes were washed in TBST before being incubated with secondary antibodies (CST). An Odyssey infrared imaging system (LI-COR Biosciences, Lincoln, USA) was employed in immunoreactive bands visualization. The analysis was conducted with ImageJ software (version 1.52).

Target prediction and luciferase reporter assay. Target gene of miR-15a-5p was predicted with TargetScan (www. targetscan.org). For establishing luciferase reporter of 3'-untranslated region (UTR) of human PHLPP2, 3'-UTR of PHLPP2 was entirely inserted into pmiR-RB-REPORT ${ }^{\mathrm{m}}$ vector (Ribobio, Guangzhou, China), downstream to renilla luciferase's coding sequence. PHLPP2 3'-UTR sequence was generated through PCR, primers were: h-PHLPP2-f (SgfI) AATGCGATCGCACCACCTGCCATTAACCCTT, h-PHLPP2-r (NotI) 662 AATGCGGCCGCCTGTCTGCCAATTCCTCCAC, h-PHLPP2-MUT-f TTACTAGTACGACGAGATGGATCTCTGTGACAG, h-PHLPP2-MUT-r GATCCATCTCGTCGTACTAGTAAAGAGAATCAA. Lipofectamine 3000 was applied in the transfection procedure. MGC803/DDP cells were transfected with vectors bearing mutant or wild-type PHLPP2 3'-UTR, together with $50 \mathrm{nM}$ precursor of miR-15a-5p (Ribobio, Guangzhou, 
China). Assays were undertaken at $48 \mathrm{~h}$ post-transfection, in which Dual-Glo ${ }^{\circledast}$ Luciferase Assay System (Promega, Madison, USA) was applied. Renilla luciferase activity was used as a normalizer.

Inclusion and exclusion criteria of patients. Inclusion criteria: patients diagnosed with gastric cancer at first reception; scheduled to undergo palliative or neoadjuvant chemotherapy with oxaliplatin; tumor not removed during the treatment; with CT baseline assessment (before the first dose of treatment); with CT re-assessment after the final dose of treatment (for patients receiving neoadjuvant therapy), or with regular CT re-assessment (for patients receiving palliative therapy); with adequate follow-up data. Exclusion criteria: chemotherapy plan changed half-way; case files not complete; follow-up interrupted; CT assessment not available; patients' rejection of inclusion.

Ethics and sample collection. Ethical approval (No: P2-162-01) was granted by the Ethics Committee of Beijing Friendship Hospital. Patients were recruited at Beijing Friendship Hospital prospectively from 1/2014 to 12/2017, with written informed consent. Blood samples were taken from patients on the morning of and before their first dose of chemotherapy. Plasma was prepared within $0.5 \mathrm{~h}$ after blood extraction. After centrifugation (10 min; $2000 \times \mathrm{g})$, the supernatant was retained and stored at $-80^{\circ} \mathrm{C}$. Clinical data were collected from patients' files.

Statistical analysis. All data were displayed as mean \pm SD, shown as representative of three independent experiments with each experiment comprising of triple replicates. Statistical analysis was conducted by a two-tailed paired Student's t-test (data analysis represented by Figure 6 is otherwise specified in its caption). A p-value $<0.05$ was considered as significant.

\section{Results}

The expression of miR-15a-p is higher in MGC803/DDP than in MGC803 cells. As shown in Figure 1A, the growth of MGC803 was significantly faster than that of MGC803/ DDP cells. Meanwhile, under the treatment of different concentrations of cisplatin, cell proliferation of MGC803 was inhibited to a much larger extent than that of MGC803/ DPP cells (Figure 1B). The IC50 of MGC803 and MGC803/ DDP was calculated to be 2.0 and $26.4 \mu \mathrm{g} / \mathrm{ml}$, respectively, revealing a Resistance Index (calculated as IC50 (MGC803/ $\mathrm{DPP}) / \mathrm{IC} 50$ (MGC803)) as 13.2. Consistently, upon the treatment with cisplatin $(2.0 \mu \mathrm{g} / \mathrm{ml})$, the apoptosis rate of MGC803 was significantly higher than that of MGC803/DDP (Figure 1C). Moreover, the expression level of miR-15a-5p in both MGC803 and MGC803/DDP cell lines was examined. Compared to MGC803 cells, miR-15a-5p was significantly upregulated in MGC803/DDP cells (Figure 1D).

miR-15a-5p enhances cisplatin-resistance of gastric cancer cells. For constructing miR-15a-5p overexpression or knockdown cell lines, we evaluated the expression of miR-15a-5p in MGC803 and MGC803/DDP cells transfected
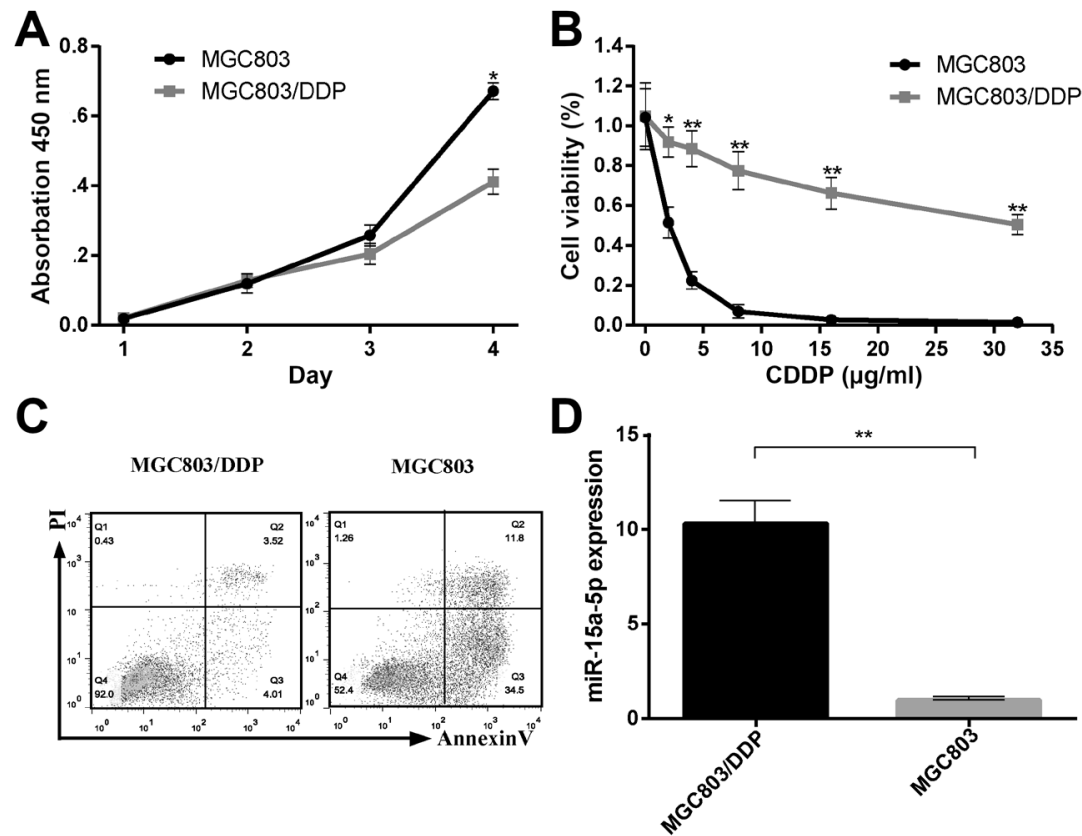

Figure 1. The expression of miR-15a-p is higher in MGC803/DDP than that in MGC803 cells. A) In the absence of cisplatin, cell proliferation of MGC803/DDP and MGC803 cells was assessed with CCK-8 assay. B) Survival of MGC803/DDP and MGC803 after 24 h treatment of increasing concentrations of cisplatin was quantified by CCK-8 assay. C) After treatment with $2 \mu \mathrm{g} / \mathrm{ml}$ cisplatin for $24 \mathrm{~h}$, the apoptosis of MGC803/DDP and MGC803 cells was measured via flow cytometry. D) qPCR detection of miR-15a-5p in MGC803/DDP and MGC803, ${ }^{\star} \mathrm{p}<0.05,{ }^{* *} \mathrm{p}<0.01,{ }^{* * * *} \mathrm{p}<0.0001$ 

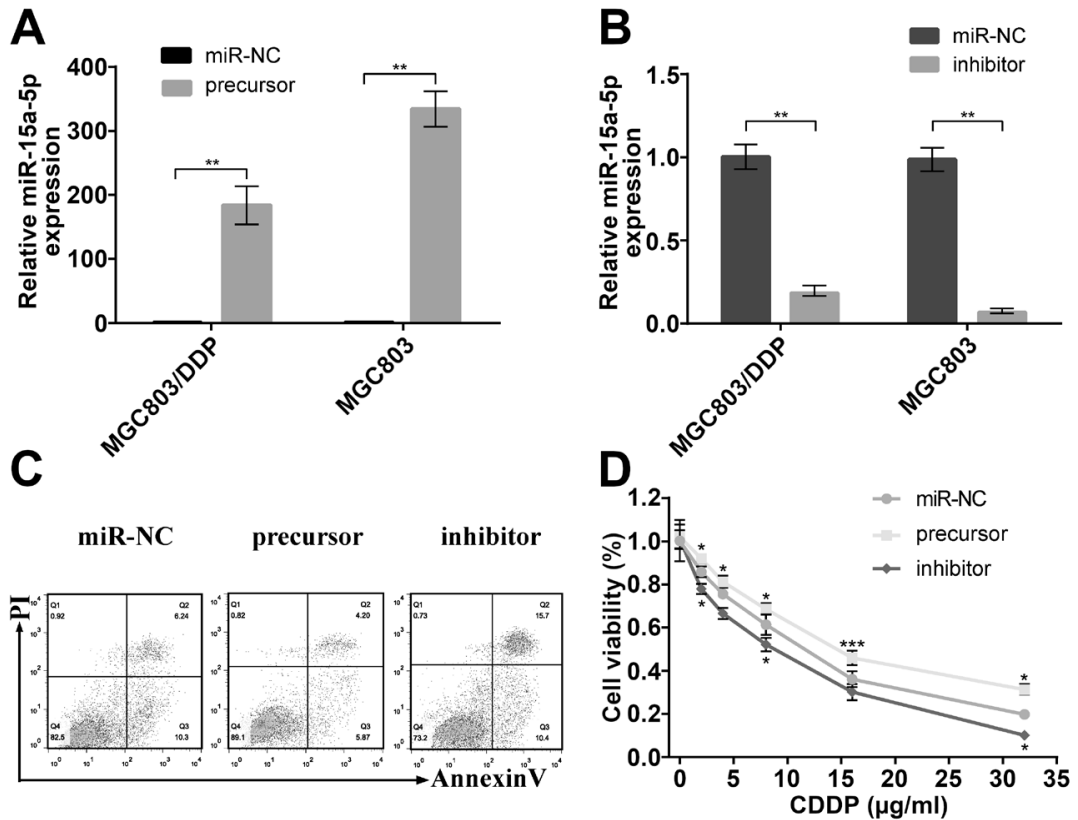

Figure 2. miR-15a-5p enhances the cisplatin-resistance of gastric cancer cells. A, B) Validation of transfection efficiency of miRNA precursor and inhibitor in MGC803/DDP and MGC803 cells by quantitative PCR. Quantification was conducted $24 \mathrm{~h}$ after transfection. U6 as an internal control. C) After treatment with $2 \mu \mathrm{g} / \mathrm{ml}$ cisplatin for $24 \mathrm{~h}$, the apoptosis rate of MGC803/DDP cells after transfection of miR-NC, miR-15a-5p precursor and inhibitor were measured by flow cytometry. D) Under the treatment of increasing concentration of cisplatin for $24 \mathrm{~h}$, the survival of MGC803/DDP cells after transfection of miR-NC, miR-15a-5p precursor, and inhibitor was measured by CCK- 8 assay. ${ }^{*} \mathrm{p}<0.05,{ }^{* * *} \mathrm{p}<0.001$ indicates significant difference compared to miR-NC group.

with miR-15a-5p precursor or inhibitor, demonstrating that miR-15a-5p expression was significantly upregulated or downregulated upon transfection of miR-15a-5p precursor or inhibitor (Figures 2A, 2B). Subsequently, we analyzed the apoptosis of the transfected cells under $2 \mu \mathrm{g} / \mathrm{ml}$ cisplatin with flow cytometry. Compared with cells transfected with miR-NC, miR-15a-5p overexpression significantly decreased cell apoptosis, whereas miR-15a-5p knockdown significantly increased cell apoptosis (Figure 2C). Afterward, we transfected another batch of MGC803/DDP cells and conducted CCK- 8 assay, revealing that miR-15a-5p overexpression significantly increased cell resistance to cisplatin of MGC803/DDP cells, whereas miR-15a-5p knockdown induced opposite effects (Figure 2D).

miR-15a-5p directly targets and inhibits PHLPP2. Targetscan database was used to predict the target genes of miR-15a-5p. PHLPP2 was selected from all the putative targets predicted by Targetscan for the significant role played by it in the proliferation and metastasis of tumor cells $[9,10]$. The predicted binding site of miR-15a-5p and PHLPP2 is shown in Figure 3A, the lower part of which displays the artificially mutated bases for subsequent dualluciferase assay. As the results of dual-luciferase assay demonstrated, compared with negative control (NC), transfection of miR-15a-5p mimic downregulated the luciferase activity of the h-PHLPP2-WT vectors. After mutating the predicted target site, the luciferase activity of the vector
A

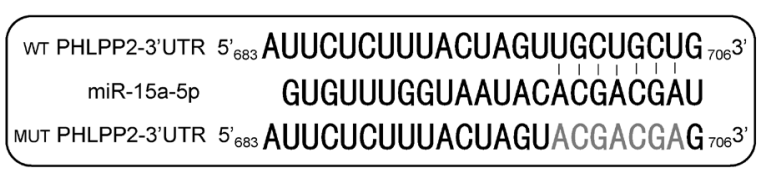

B

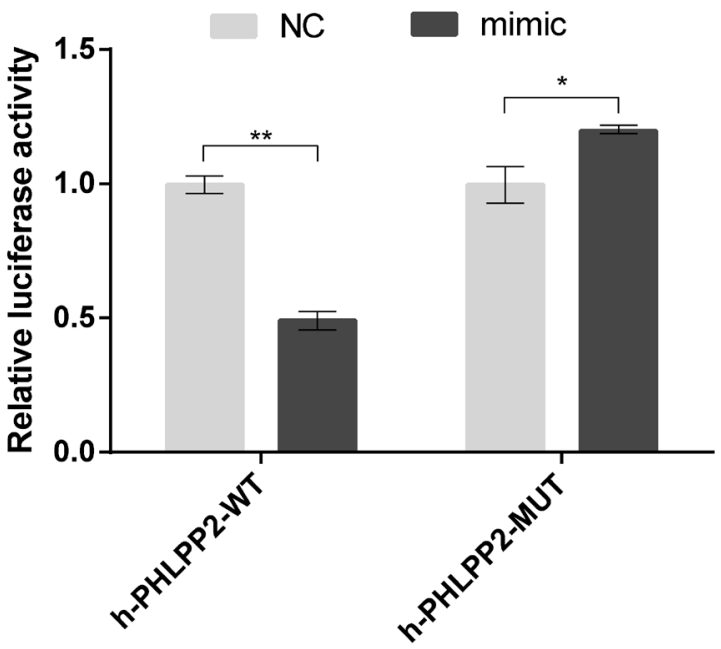

Figure 3. miR-15a-5p directly targets and inhibits PHLPP2. A) Targetscan-predicted binding site of miR-15a-5p and PHLPP2, and artificial mutated bases. B) Luciferase reporter assay was performed in MGC803/ DDP cells to detect the relative luciferase activities of WT and mutated PHLPP2 reporters. Renilla luciferase vector was used as an internal control. ${ }^{*} \mathrm{p}<0.05,{ }^{* *} \mathrm{p}<0.01$ 
h-PHLPP2-MUT has a significant reversion (Figure 3B). These outcomes indicated that miR-15a-5p may directly interact and regulate the expression of PHLPP2 through the predicted binding site.

Further validation of PHLPP2 as a target of miR-15a-5p via $\mathrm{qPCR}$ and western blot. To further validate the regulation of miR-15a-5p on PHLPP2, we firstly measured the expression of PHLPP2 on mRNA level in both MGC803/ DDP and MGC803 cells and found that PHLPP2 showed significantly lower expression in MGC803/DDP cells (Figure 4A), in which miR-15a-5p is highly expressed as previously demonstrated in Figure 1D. Consistently, the downregulated protein level of PHLPP2 in MGC803/DPP cells was also observed by western blotting (Figure 4B). In addition, it was further demonstrated that overexpression or knockdown of miR-15a-5p could decrease or increase the expression of PHLPP2 in both MGC803/DDP and MGC803 cells (Figures 4C, 4D). Considering the ability of PHLPP2 to regulate AKT pathway, phosphorylation of AKT, as well as activity of the AKT pathway was evaluated by western blotting. The results showed that phosphorylation of AKT was promoted in MGC803/DPP cells relative to MGC803 cells, and in miR-15a-5p overexpressed cells relative to NC cells, while inhibited in miR-15a-5p knockdown cells (Figures 4B, 4D). Collectively, the expression of miR-15a-5p was negatively related to PHLPP2 expression and positively related to AKT activity.

PHLPP2 overexpression decreases resistance to cisplatin while miR-15a-5p overexpression partially reverses the change. We transfected MGC803/DDP cells in three manners: 1 ) with empty vectors + miR-NC; 2 ) with vectors overexpressing PHLPP2 + miR-NC; 3 ) with vectors overexpressing PHLPP2 + miR-15a-5p precursor. Flow cytometry was conducted to assess the apoptosis of the three batches of differently transfected cells under $2 \mu \mathrm{g} / \mathrm{ml}$ of cisplatin. The results demonstrated that overexpressing PHLPP2 significantly increased cell apoptosis while the transfection of miR-15a-5p precursor partially restored the alteration (Figure 5A). Also, we conducted CCK-8 assay to measure the survival of transfected cells under different concentrations
A

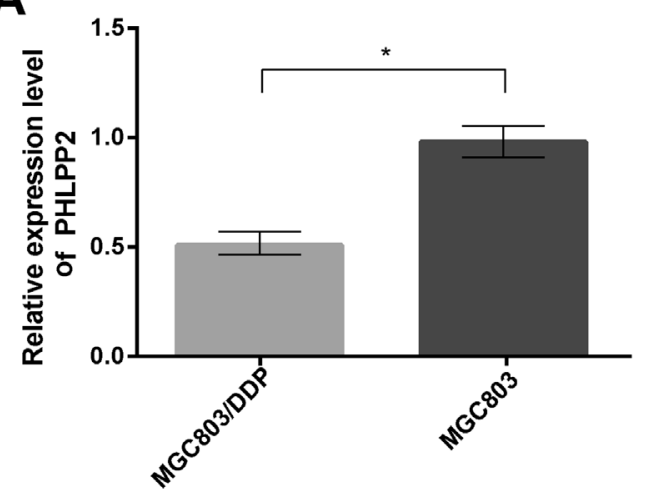

C

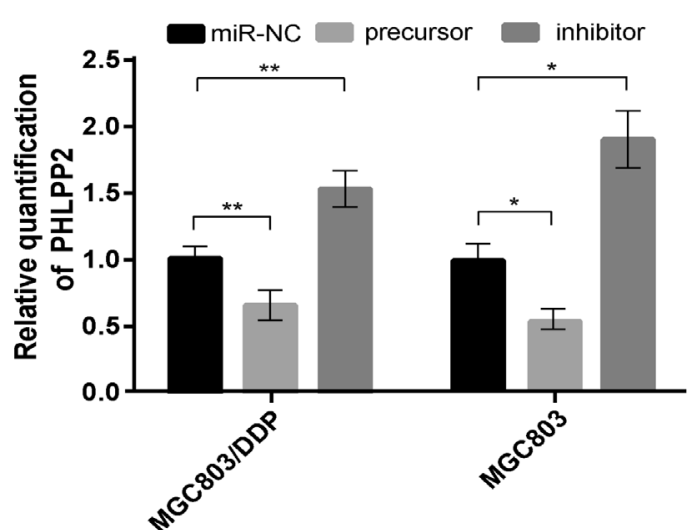

B

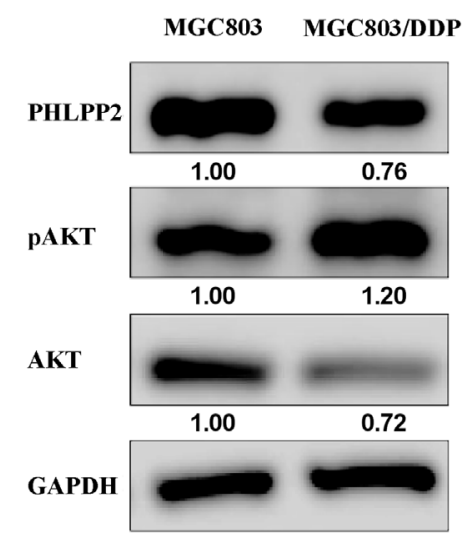

D

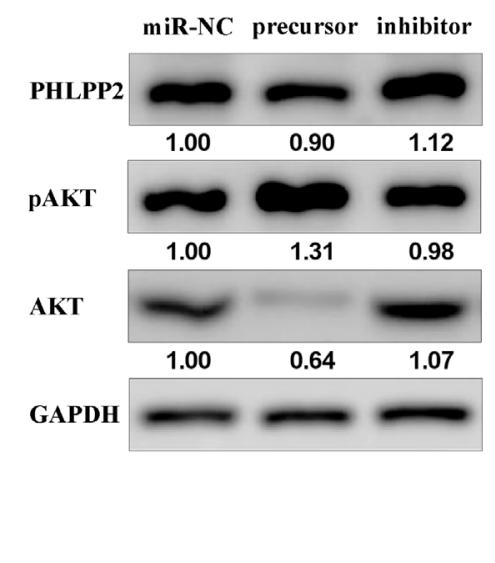

Figure 4. Further validation of PHLPP2 as a target of miR-15a-5p via qPCR and western blot. A) qPCR quantification of PHLPP2 in MGC803/DDP and MGC803 on the mRNA level. B) Profiles of PHLPP2 and phosphorylation of Akt in MGC803 and MGC803/DDP cells on the protein level. GAPDH as an internal control. C) Quantification of mRNA of PHLPP2 in MGC803/DDP and MGC803 cells, $24 \mathrm{~h}$ after transfection of miR-NC, miR-15a-5p precursor, and inhibitor, by quantitative PCR. GAPDH as an internal control. D) Western blot assessment of PHLPP2 and phosphorylation of Akt in MGC803/DDP cells, $24 \mathrm{~h}$ after transfection of miR-NC, miR-15a-5p precursor and inhibitor. GAPDH as an internal control. ${ }^{\star} \mathrm{p}<0.05,{ }^{\star *} \mathrm{p}<0.01$ 
of cisplatin and found that overexpressing PHLPP2 significantly decreased cisplatin resistance while transfection of miR-15a-5p precursor partially restored the alteration as well (Figure 5B).

Serum miR-15a-5p level is significantly correlated to patients' response to chemotherapy and survival. Given the functions of miR-15a-5p revealed by in vitro study, serum miR-15a-5p level of a total of 82 patients was quantified via

A vector + miR-NC PHLPP2 + miR-NC PHLPP2 + precursor
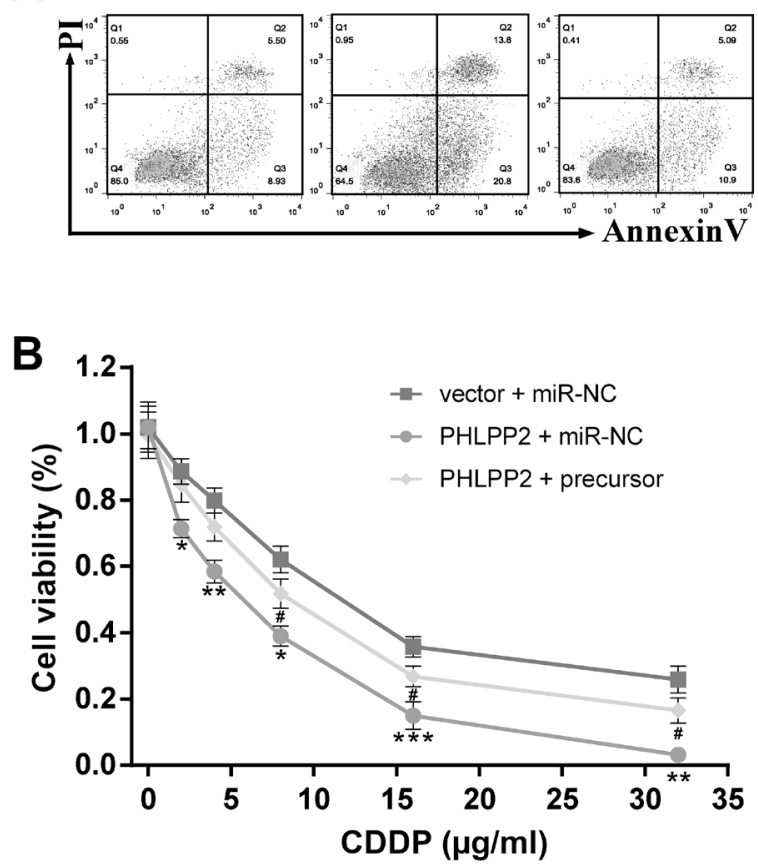

Figure 5. PHLPP2 overexpression decreases resistance to cisplatin while miR-15a-5p overexpression partially reverses the change. A) After treatment with $2 \mu \mathrm{g} / \mathrm{ml}$ cisplatin for $24 \mathrm{~h}$, the apoptosis of MGC803/DDP cells after transfection of: 1) empty vectors + miR-NC; 2 ) vectors overexpressing PHLPP2 + miR-NC; 3) vectors overexpressing PHLPP2 + miR-15a-5p precursor were measured by flow cytometry. B) Under $24 \mathrm{~h}$ treatment of increasing concentrations of cisplatin, the survival of MGC803/DDP cells after transfection in the same manner as above was measured by CCK-8 assay. ${ }^{*} \mathrm{p}<0.05,{ }^{* *} \mathrm{p}<0.01,{ }^{* * *} \mathrm{p}<0.001$ indicates significant difference compared to vector + miR-NC group; ${ }^{\sharp} \mathrm{p}<0.05$ indicates significant difference compared to PHLPP2 + miR-NC group.
qPCR. The resulting quantities of patients' serum miR-15a-5p level were labeled low or high with the median as a cut-off line. Then patients were categorized into two groups $(\mathrm{CR} / \mathrm{PR}$ group and $\mathrm{SD} / \mathrm{PD}$ group) according to their chemotherapy response status (assessed by CT scan, according to RECIST 1.1 criteria [11]). We found that patients with better response to oxaliplatin (the CR/PR group) have significantly lower serum miR-15a-5p level (Figure 6A). Then a survival analysis with a log-rank test was conducted with patients' follow-up data. The results indicated that lower serum miR-15a-5p level is significantly correlated with better survival ( $<<0.05$, Figure 6B).

\section{Discussion}

miR-15a-5p participates in the regulation of cell proliferation and apoptosis in several pathways. Previous research suggested that miR-15a-5p is frequently downregulated in various types of carcinoma [12-16]. miR-15a-5p is also significantly underexpressed in primary multiple myeloma and participated in its tumorigenesis with VEGF-A as a target molecule [17]. Additionally, it was reported that decreased miR-15-5p in cancer-associated fibroblasts (CAFs) promotes growth and invasiveness of tumors by reducing its inhibition of Fgf2 and Fgfr1 [13]. On the other hand, there is another study suggesting that miR-15a overexpression inhibits cell growth, suppresses migration, and arrests cell cycle at G1 yet does not enhance apoptosis of breast cancer [18]. However, the relation between cell cycle arrest and chemoresistance is too complicated to elaborate in this article.

Akt regulates cell survival directly through suppressing various pro-apoptotic signals. PHLPP2 is a phosphatase that is capable of dephosphorylating pAkt [19]. Reduced PHLPP2 expression will result in a prolonged Akt phosphorylation, which in turn will activate assorted downstream targets, promoting the growth and survival of cells. In other words, as a member of the PHLPP family, PHLPP2 suppresses PI3K/ AKT pathway, thus enhancing apoptosis in various cancer types [20]. In prostate cancer, previous studies showed that the frequency of PHLPP2 depletion is even rivaling that of PTEN [19]. In this research, our findings suggested that in gastric cancer cell line MGC803, PHLPP2 is a direct target of
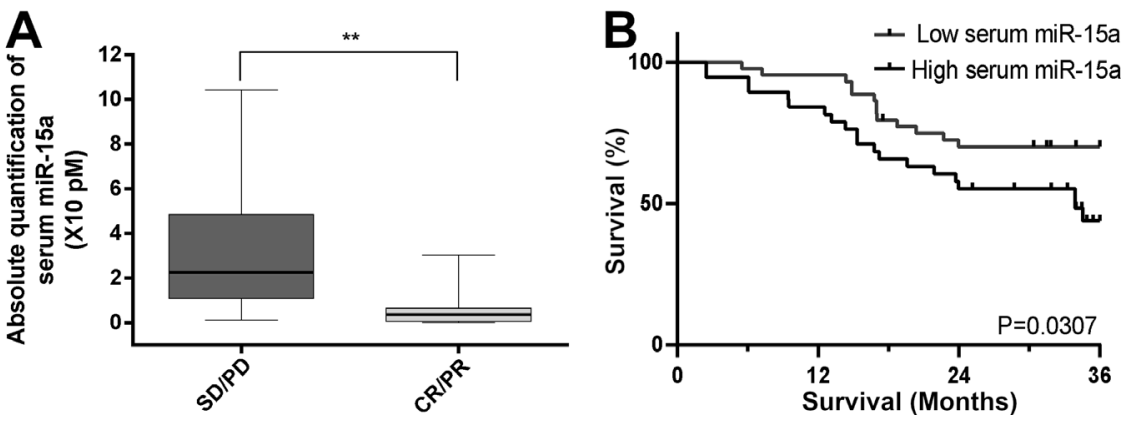

Figure 6. Serum miR-15a-5p level is significantly correlated to patients' responses to chemotherapy and long-term survival. A) Absolute quantification of serum miR-15a-5p levels in 82 gastric cancer patients, all of whom underwent neo-adjuvant or salvage oxaliplatin-based chemotherapy. Blood was extracted before the first dose of chemotherapy. CT evaluation for change of tumor volume was conducted according to RECIST1.1 criteria. B) Survival analysis of serum miR-15a-5p in the same 82 gastric cancer patients. Total time of follow-up: 36 months. ${ }^{* *} \mathrm{p}<0.01$ 
miR-15a-5p. Even with the presence of cisplatin, overexpression of miR-15a-5p significantly antagonizes the inhibitory effects of PHLPP2 on cell survival.

In this study, we demonstrated that miR-15a-5p regulates MGC803 cells' sensitivity to cisplatin by targeting PHLPP2. Also, we experimented with patients' serum and found that patients with higher serum miR-15a-5p levels generally have a significantly worse response to oxaliplatin-based chemotherapy and worse 3-year survival. Since cells with higher miR-15a-5p level are more resistant to cisplatin, can we take it for granted that higher serum level of miR-15a-5p is correlated with worse response to oxaliplatin-based chemotherapy as well? In other words, does a higher intracellular level of a certain miRNA dictate a higher extracellular level of itself? Regarding how extracellular miRNAs, or specifically serum miRNAs, come to be in the first place and stay remarkably stable, there are currently five major hypotheses: 1) through exosomes; 2) through microvesicles other than exosomes, namely ectosomes and microparticles; 3 ) through apoptotic bodies; 4) through high density lipoprotein; 5) through protein complex [7]. So the question becomes: on the same patient, does the circulating level of a certain miRNA reflect its level in the tumor? There're two opposite opinions in the field: one suggests that tumor cells shed miRNAs passively into circulation when they die [21]; the other suggests that in breast cancer cells at least, tumor cells actively and selectively secret miRNAs into circulation [22]. Moreover, there is also a direct answer to the question above: the research of Cookson et al. suggested that for some miRNAs, their circulating level directly reflects their level in the tumor; but for other miRNAs, their circulating level does not [23].

In conclusion, miR-15a-5p was observed to be higher expressed in the cisplatin-resistant MGC803/DDP cell line, which could affect the cisplatin sensitivity of gastric cancer cells by promoting cell survival and reducing apoptosis. It functions by directly binding and inhibiting PHLPP2. This study provides solid evidence that miR-15a-5p could serve as a potential target in the treatment of gastric cancer, and that serum miR-15a-5p level could serve as a promising biomarker to predict patients' response to oxaliplatin-based chemotherapy.

Acknowledgments: This work was supported by a grant from the Municipal Natural Science Foundation of Beijing (17G10203).

\section{References}

[1] TORRE LA, BRAY F, SIEGEL RL, FERLAY J, LORTETTIEULENT J et al. Global cancer statistics, 2012. CA Cancer J Clin 2015; 65: 87-108. https://doi.org/10.3322/caac.21262

[2] RABIK CA, DOLAN ME. Molecular mechanisms of resistance and toxicity associated with platinating agents. Cancer Treat Rev 2007; 33: 9-23. https://doi.org/10.1016/j. ctrv.2006.09.006
[3] COCCONI G, CARLINI P, GAMBONI A, GASPERONI S, RODINO C et al. Italian Oncology Group for Clinical Research. Cisplatin, epirubicin, leucovorin and 5-fluorouracil (PELF) is more active than 5-fluorouracil, doxorubicin and methotrexate (FAMTX) in advanced gastric carcinoma. Ann Oncol 2003; 14: 1258-1263. https://doi.org/10.1093/annonc/ $\operatorname{mdg} 329$

[4] WEBB A, CUNNINGHAM D, SCARFFE JH, HARPER P, NORMAN A et al. Randomized trial comparing epirubicin, cisplatin, and fluorouracil versus fluorouracil, doxorubicin, and methotrexate in advanced esophagogastric cancer. J Clin Oncol 1997; 15: 261-267. https://doi.org/10.1200/ JCO.1997.15.1.261

[5] BARTEL DP. MicroRNAs: genomics, biogenesis, mechanism, and function. Cell 2004; 116: 281-297. https://doi. org/10.1016/s0092-8674(04)00045-5

[6] LAGOS-QUINTANA M, RAUHUT R, LENDECKEL W, TUSCHL T. Identification of novel genes coding for small expressed RNAs. Science 2001; 294: 853-858. https://doi. org/10.1126/science. 1064921

[7] MAHMODUL HS. Extracellular/Circulating MicroRNAs: Release Mechanisms, Functions and Challenges. Achievements in the Life Sciences 2016; 10: 175-186. https://doi. org/10.1016/j.als.2016.11.007

[8] BROGNARD J, SIERECKI E, GAO T, NEWTON AC. PHLPP and a second isoform, PHLPP2, differentially attenuate the amplitude of Akt signaling by regulating distinct Akt isoforms. Mol Cell 2007; 25: 917-931. https://doi. org/10.1016/j.molcel.2007.02.017

[9] MOLINA JR, AGARWAL NK, MORALES FC, HAYASHI Y, ALDAPE KD et al. PTEN, NHERF1 and PHLPP form a tumor suppressor network that is disabled in glioblastoma. Oncogene 2012; 31: 1264-1274. https://doi.org/10.1038/ onc. 2011.324

[10] LI X, STEVENS PD, LIU J, YANG H, WANG W et al. PHLPP is a negative regulator of RAF1, which reduces colorectal cancer cell motility and prevents tumor progression in mice. Gastroenterology 2014; 146: 1301-12.e1-10. https://doi. org/10.1053/j.gastro.2014.02.003

[11] EISENHAUER EA, THERASSE P, BOGAERTS J, SCHWARTZ LH, SARGENT D et al. New response evaluation criteria in solid tumors: revised RECIST guideline (version 1.1). Eur J Cancer 2009; 45: 228-247. https://doi. org/10.1016/j.ejca.2008.10.026

[12] SAMPATH D, LIU C, VASAN K, SULDA M, PUDUVALLI VK et al. Histone deacetylases mediate the silencing of miR15a, miR-16, and miR-29b in chronic lymphocytic leukemia. Blood 2012; 119: 1162-1172. https://doi.org/10.1182/ blood-2011-05-351510

[13] MUSUMECI M, COPPOLA V, ADDARIO A, PATRIZII M, MAUGERI-SACCÀ $M$ et al. Control of tumor and microenvironment cross-talk by miR-15a and miR-16 in prostate cancer. Oncogene 2011; 30: 4231-4242. https://doi. org/10.1038/onc.2011.140

[14] CAI CK, ZHAO GY, TIAN LY, LIU L, YAN K et al. miR-15a and miR-16-1 downregulate CCND1 and induce apoptosis and cell cycle arrest in osteosarcoma. Oncol Rep 2012; 28: 1764-1770. https://doi.org/10.3892/or.2012.1995 
[15] DINIZ MG, GOMES CC, DE CASTRO WH, GUIMARÃES AL, DE PAULA AM et al. miR-15a/16-1 influences BCL2 expression in keratocystic odontogenic tumors. Cell Oncol (Dordr) 2012; 35: 285-291. https://doi.org/10.1007/s13402012-0087-3

[16] YANG J, CAO Y, SUN J, ZHANG Y. Curcumin reduces the expression of Bcl-2 by upregulating miR-15a and miR-16 in MCF-7 cells. Med Oncol 2010; 27: 1114-1118. https://doi. org/10.1007/s12032-009-9344-3

[17] SUN CY, SHE XM, QIN Y, CHU ZB, CHEN L et al. miR-15a and miR-16 affect the angiogenesis of multiple myeloma by targeting VEGF. Carcinogenesis 2013; 34: 426-435. https:// doi.org/10.1093/carcin/bgs333

[18] LUO Q, LI X, LI J, KONG X, ZHANG J et al. MiR-15a is underexpressed and inhibits the cell cycle by targeting CCNE1 in breast cancer. Int J Oncol 2013; 43: 1212-1218. https://doi. org/10.3892/ijo.2013.2034

[19] NEWTON AC, TROTMAN LC. Turning Off AKT: PHLPP as a Drug Target. Annu Rev Pharmacol Toxicol 2014; 54: 537-558. https://doi.org/10.1146/annurev-pharmtox-011112-140338
[20] O’NEILL AK, NIEDERST MJ, NEWTON AC. Suppression Of survival signaling pathways by the phosphatase PHLPP. FEBS J 2013; 280: 572-583. https://doi.org/10.1111/j.17424658.2012.08537.x

[21] BRASE JC, WUTTIG D, KUNER R, SULTMANN H. Serum microRNAs as non-invasive biomarkers for cancer. Mol Cancer 2010; 9: 306. https://doi.org/10.1186/1476-4598-9306

[22] PIGATI L, YADDANAPUDI SC, IYENGAR R, KIM DJ, HEARN SA et al. Selective release of microRNA species from normal and malignant mammary epithelial cells. PLoS One 2010; 5: e13515. https://doi.org/10.1371/journal. pone.0013515

[23] COOKSON VJ, BENTLEY MA, HOGAN BV, HORGAN K, HAYWARD BE et al. Circulating microRNA profiles reflect the presence of breast tumours but not the profiles of microRNAs within the tumors. Cell Oncol (Dordr) 2012; 35: 301-308. https://doi.org/10.1007/s13402-012-0089-1 